\title{
Ensayo de ladrillos a tracción
}

El proyecto estructural de fábrica de ladrillo, cuando se basa en el vigente British Standard Code of Practice III (1), exige un conocimiento de la resistencia del ladrillo y. las proporciones de mortero.

No puede establecerse con precisión la relación que existe entre la resistencia a compresión del mortero y la de la pared en que se usa, pero se ha sugerido que un cambio en la resistencia de la pared se relaciona con la raíz tercera o cuarta del cambio en la resistencia del mortero. Para un cambio en la resistencia del ladrillo parece ser más crítica esta relación, siendo aproximadamente proporcional a la raíz cuadrada.

Cuando la fábrica de ladrillo se somete a condiciones de carga de rotura en un panel de ensayo, la rotura se produce inicialmente por resquebrajamiento vertical, sometiendo el gran esfuerzo lateral del mortero a los ladrillos individuales a unas condiciones de esfuerzo complejas que es predominantemente a tracción. Con las condiciones de carga a rotura in mente se decidió investigar alguna de las propiedades a tracción del ladrillo, ya que se consideró que podía establecerse una relación más directa empleando un ensayo más semejante a la forma de rotura que el llamado ensayo de resistencia a compresión.

\section{Los ensayos investigados fueron:}

a) Tracción indirecta de ladrillos enteros.

b) Tracción indirecta de probetas de ladrillo.

c) Tracción directa de ladrillos enteros y cortados.

d) Tracción directa del ladrillo empleando un sistema de cuña expansiva.

e) Tensión por flexión de ladrillos enteros (módulo de rotura).

f) Resistencia a compresión entre placas de madera contrachapada.

g) Resistencia a compresión entre placas con cepillo (cepillos Hilsdorf).

\section{ENSAYO DE TRACCION INDIRECTA: LADRILLOS}

El ensayo de tracción indirecta o brasileño se halla bien establecido en la tecnología del hormigón, pero no se ha aplicado previamente al ensayo de ladrillos hasta que los 
autores han tenido noticia de ello. El ensayo se llevó a cabo sobre ambos ejes principales del ladrillo, y se emplearon tiras de madera contrachapada para superar el peligro de caída prematura debida a las irregularidades superficiales (figs. 1 y 2).

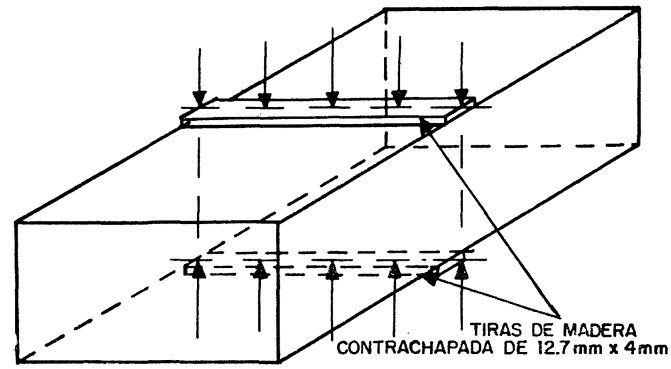

Fig. 1.-Ensayo de tracción indirecta: rotura de ladrillo a lo ancho.

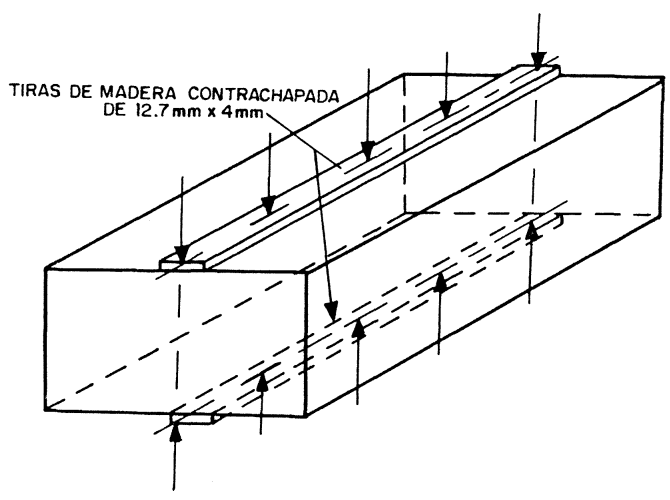

Fig. 2.-Ensayo de tracción indirecta: rotura de ladrillo a lo largo.

Se han ensayado distintas variedades de ladrillo, tanto macizos como perforados, y los resultados se han referido en otras ocasiones (2) y (3).

El método produjo resultados más elevados como puede esperarse cuando las unidades sết rompen a través de su ancho. Es fácil de llevar a cabo, emplea mucho menos materíal de sujeción que el ensayo a compresión y consume menos tiempo en su ejecución.

La velocidad de carga dentro de los márgenes ensayados, 1,379 a $18,134 \mathrm{MN} / \mathrm{m}^{2} / \mathrm{min}$, no afecta el valor de la resistencia a tracción indirecta, distinto a lo que ocurre con el ensayo a compresión, donde la fijación de los platos de la máquina y/o de los cartones duros o contrachapados de relleno afecta la resistencia final registrada.

El tipo de material de relleno empleado afecta la resistencia a tracción indirecta registrada, debiéndose emplear un material de relleno standard; esto también se aplica al ensayo a compresión, pero la norma (4) permite el empleo de cualquier tipo de madera contrachapada.

La relación resistencia a tracción/resistencia a compresión para este ensayo, expresada como porcentaje, oscila de 7,92 a 14,16 .

El ensayo es más difícil de interpretar si se emplea una unidad multiperforada; los ladrillos acanalados deben rellenarse con mortero antes de su ensayo.

\section{ENSAYO DE TRACCION INDIRECTA: PROBETAS-TESTIGO DE LADRILLO}

Para comprobar los valores obtenidos con los ladrillos enteros, se cortaron testigos, de $43 \mathrm{~mm}$ de diámetro, de los laterales y del centro de los ladrillos de arcilla cocida, comparando los resultados. También se tomaron testigos de ladrillos silicocalcáreos; como éstos son unidades macizas es posible comparar muestras tomadas en los tres ejes.

Las muestras tomadas del borde de los ladrillos de arcilla cocida dieron resultados más bajos que las tomadas en el centro debido al procedimiento de fabricación: el proceso 
de extrusión necesita que la arcilla pase a través de una boquilla que crea una laminación espiral en el ladrillo crudo (fig. 3). Esto significa que la resistencia a tracción puede ser nominalmente diferente en los dos ejes. Este fenómeno también se notó en el ensayo de tracción directa.

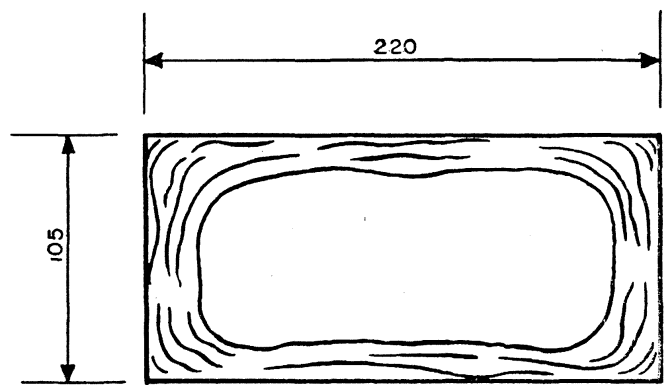

Fig. 3.-Posible esquema de laminación en ladrillos extrusionados.

Los testigos sacados del lado y del centro de los ladrillos silicocalcáreos dieron resultados notablemente iguales, dando unos valores medios de 2,827 y $2,813 \mathrm{MN} / \mathrm{m}^{2}$, respectivamente. Una diferencia notoria en el ensayo de tracción indirecta se observó entre los testigos cortados en la cara-base de los ladrillos, dando una media de $2,6 \mathrm{MN} / \mathrm{m}^{2}$. También esta diferencia puede explicarse refiriéndose a la fabricación. Los ladrillos silicocalcáreos ensayados en el experimento se prensaron sobre la cara de $21,8 \times 6,70 \mathrm{~cm}$, lo que automáticamente afecta la orientación de los granos de arena y produce una pieza que tiene una resistencia ligeramente diferente en cada uno de los ejes. Además, como el material está prensado en el molde, los efectos friccionales de los costados aseguran que el material en la cara superior esté sometido a una presión más elevada que en la cara-base, con lo que resulta que el ladrillo es nominalmente más fuerte junto al borde

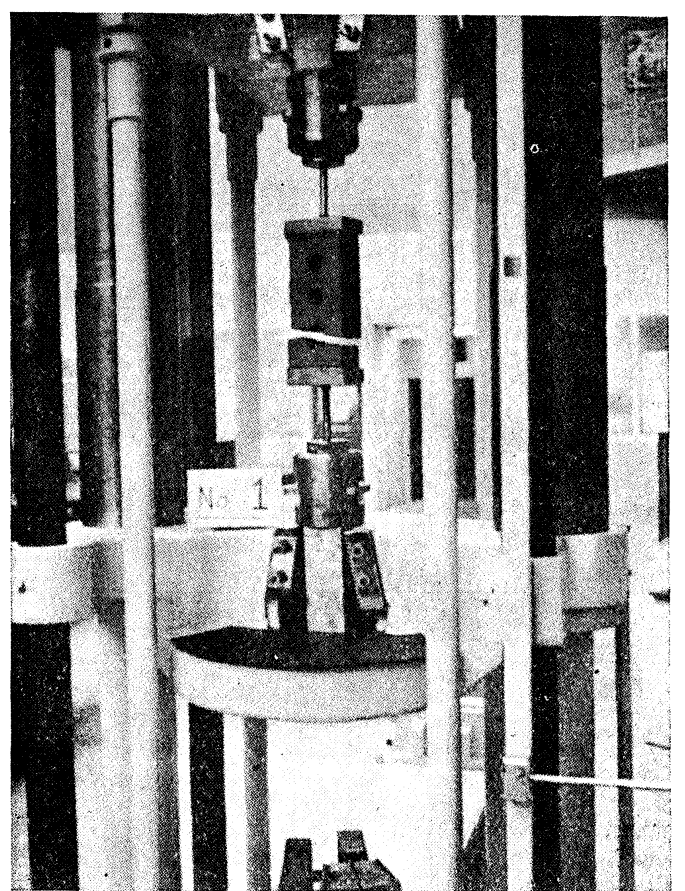

Fig. 4.-Ensayo de tracción directa. prensado. Para prevenir una rotura prematura de los testigos durante el ensayo, se emplearon placas de cartón duro o madera contrachapada $(1,7 \times 9,5 \mathrm{~mm})$ entre los testigos y los platos de la prensa.

\section{ENSAYO DE TRACCION DIRECTA PARA LADRILLOS ENTEROS Y CORTADOS}

El ensayo de tracción directa se llevó a cabo en varios tipos de ladrillos, pegando placas de hierro dulce estriadas a las bases de la probeta mediante una resina epoxi con un filler. Las mordazas Denison standard de 1/2 pulgada de diámetro se atornillaron al extremo de los platos y se ensayaron las probetas en una máquina de 50 toneladas de capacidad (fig. 4). Los primeros resultados indicaron que este método de ensayo no daría la resistencia a tracción real de un ladrillo de arcilla hecho por extrusión, por las razones previamente mencionadas, pero el método es satisfactorio para los ladrillos prensados.

Intentando superar la dificultad de una rotura prematura en los bordes de los ladrillos extrusionados, se hicieron ensayos sobre probetas a las que se había cortado aproximadamente una pulgada en cada lado, teniendo cuidado de que quede al menos una longitud 
de ladrillo de unos $13 \mathrm{~cm}$ para eliminar las dificultades de fijación en los extremos debido a las placas, como describió Spetla y Kadlecek (5). Los ladrillos cortados dieron resultados más favorables, pero aún éstos eran de un orden más bajo que los valores de tracción indirecta. Esto no se consideraba realístico para hacer una comparación sobre esta base, ya que el ensayo directo permite que la rotura ocurra en la posición más débil y la excentricidad accidental no puede fácilmente tenerse en cuenta, mientras que el ensayo de tracción indirecta mide la resistencia a tracción en una posición específica a través o a lo largo del ladrillo.

\section{ENSAYO DE TRACCION DIRECTA EM- PLEANDO UN SISTEMA DE MACHOS DE EXPANSION}

Como se encontraron numerosas dificultades con el ensayo de tracción directa aplicado axialmente se desarrolló un sencillo "sistema de machos de expansión" en un intento de medir la resistencia a tracción en una posición específica del ladrillo.

El sistema de machos expansionadores (fig. 5) consiste en dos machos con bordes afilados que entran en dos huecos de un cilindro partido con los extremos interiores en la misma forma de cono.

Para determinar la resistencia a tracción media del ladrillo se hace un agujero de $32 \mathrm{~mm}$ de diámetro a través del centro de la cara de base, y el cilindro partido se coloca en el agujero. Entonces, se colocan los machos en los extremos del cilindro y se aplica una carga vertical a dichos machos con una máquina de ensayo a compresión. De la carga a rotura registrada, se calcula la componente horizontal.

Se intentó el calibrado de este sistema empleando una disposición de anillo de prueba, pero esto resultó difícil. La medida de las cargas vertical y horizontal calibrada era posible supuesto que el cilindro partido se mantenía en la posición teóricamente correcta al final de los machos para apoyar en la máxima área de contacto. Si el cilindro partido se fuerza por encima de este punto, aparecía un acolchonamiento en los machos y resultaban lecturas falsas.

En el ensayo real del ladrillo el cilindro partido quedaba sujeto por el agujero en el ladrillo y se consideraba que la componente horizontal de la carga vertical, determinada teóricamente, es suficientemente exacta para fines prácticos. 
En conjunción con este sistema del autor, McNicholas examinó esta técnica fotoelásticamente, dándose una descripción de su trabajo en otro lugar (6). El esfuerzo de tracción directa obtenido empleando el sistema de machos de expansión se calcula como un valor medio, mientras que McNicholas demuestra que aparece un factor de concentración de esfuerzos de 4 junto al borde del agujero en la rotura, lo que implica una falta de linealidad en la relación tensión/deformación.

\section{ENSAYO DE TRACCION POR FLEXION}

Este ensayo se llevó a cabo empleando tres redondos de hierro dulce de $19 \mathrm{~mm}$ de diámetro $\times 15 \mathrm{~cm}$ de longitud, tal como indica la figura 6 . El redondo superior se colocó en el centro del ladrillo y los redondos de la base a $18 \mathrm{~cm}$ en puntos equidistantes de

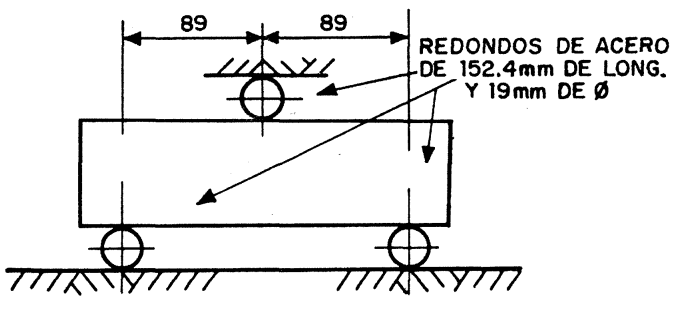

Fig. 6.-Ensayo de tracción por flexión. la línea central, aplicándose la carga en una máquina de ensayo a compresión. Como era de esperar, este ensayo dio resultados extremadamente variables y no se señaló ninguna tendencia o relación definida.

\section{ENSAYO A COMPRESION ENTRE PLACAS DE CARTON O MADERA CONTRACHAPADA}

Los ensayos a compresión se llevaron a cabo de acuerdo con la norma (4) y se emplearon como dato para todos los demás ensayos. También se hizo una comparación entre el ensayo en húmedo y en seco.

\section{ENSAYO A COMPRESION ENTRE PLACAS CON CEPILLO (CEPILLO HILSDORF)}

Cuando se ensayan ladrillos a compresión, el material cerámico o silicocalcáreo adyacente a los platos de carga se encuentran sujetos lateralmente. Para eliminar este efecto externo se proyectaron y construyeron un par de placas con cepillo para tener una rigidez similar a los cepillos empleados por Grimer (7) en la Building Research Station para el ensayo de hormigón. La figura 7 ilustra los cepillos que constan de cerdas de acero plateado de $0,8 \mathrm{~cm}^{2}$ separadas por planchas de $0,05 \mathrm{~mm}$. La cabeza del cepillo estaba enmarcada por placas de acero de $25 \mathrm{~mm}$ de ancho $\times 38 \mathrm{~mm}$ de altura colocadas a inglete y atornilladas a la base o placa de cabeza. Las unidades de ensayo que emplean este medio evitan que se ejerza cualquier esfuerzo cortante transversal apreciable en la interfase de la muestra y las placas-cepillo.

El ensayo de ladrillos de arcilla cocida entre cepillos Hilsdorf no puede llevarse a cabo felizmente sin el tratamiento de las caras de apoyo para compensar los efectos de las irregularidades superficiales, empleándose con este fin una película de escayola por considerarlo como el método más barato asequible a los autores.

Si se ensayan ladrillos silicocalcáreos, la película de yeso sería innecesaria por su uniformidad y textura.

Al ensayar ladrillos de esta manera ocurre la rotura a tracción completa de la pieza y se 
encuentra una relación estrecha entre estos resultados y el ensayo de tracción indirecta para unidades partidas a lo ancho.

\section{ENSAYO COMPARATIVO DE PILARES DE FABRICA DE LADRILLO}

A fin de estudiar los efectos de la sujeción de placas sobre una pila de altura variable de fábrica de ladrillo, se hicieron ensayos de comparación sobre pilares construidos con morteros 1:3, 1:6 y 1:12 y también sobre pilares de ladrillos rejuntados en seco.

Se obtuvieron dos series de resultados: una colocando las muestras entre placas de cartón o madera contrachapada y la otra entre los cepillos Hilsdorf.

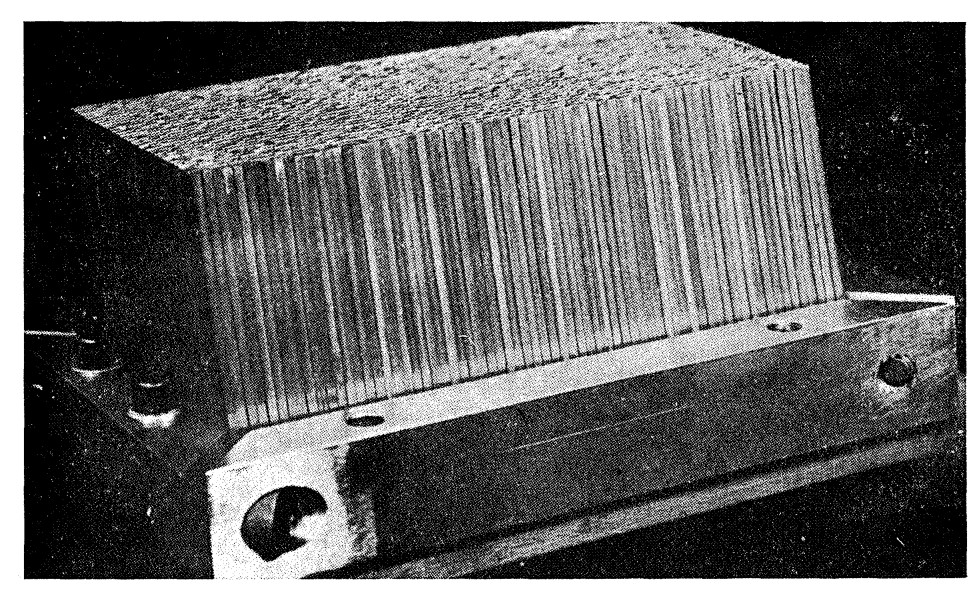

Fig. 7.-Cepillo Hilsdorf.

Los ladrillos usados en este experimento eran silicocalcáreos, y se eligió una gama de morteros más amplia de las utilizadas normalmente con la esperanza de que pudieran obtenerse resultados perfectamente definidos. Para evitar resultados enmascarados se decidió no emplear cal en el mortero, aunque se apreció que haciéndolo así se reduce la trabajabilidad del mortero e, indudablemente, se afecta la trabazón entre el ladrillo y el mortero.

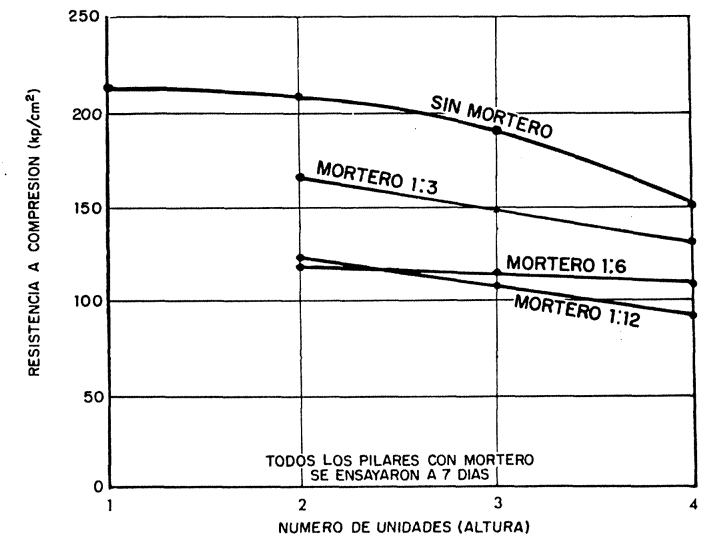

Fig. 8.-Pilares ensayados entre cepillos Hilsdorf.

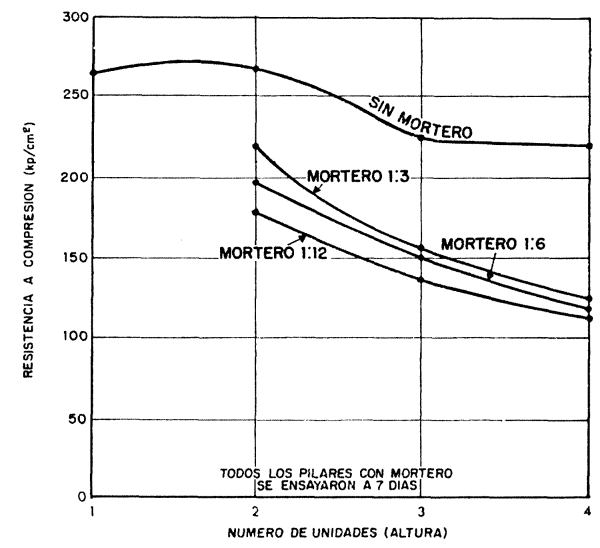

Fig. 9.-Pilares ensayados entre placas de madera contrachapada de caoba. 
Las figuras 8 y 9 ilustran gráficamente los resultados obtenidos e indican: que en la serie de pilares trabados con mortero ensayados, los estudiados con los cepillos Hilsdorf dieron una relación lineal, mientras que las muestras ensayadas entre placas de cartón o madera no.

Los esquemas de rotura para las probetas ensayadas entre placas de cepillo Hilsdorf eran generalmente similares, fluyendo el mortero hasta o próximo a la carga final acompañado del hendimiento vertical de los ladrillos. Las muestras ensayadas entre placas de cartón o madera cayeron por hendimiento transversal y/o vertical de las unidades, fluyendo el mortero como en las otras muestras. Algunas de las parejas de ladrillos rompieron a $45^{\circ}$, exhibiendo la llamada rotura cortante debida a la sujeción de las placas.

\section{MEDIDAS DE DEFORMACIONES EN PILARES}

Las medidas de las deformaciones se efectuaron sobre un pilar construido con mortero 1:3 y ensayado inicialmente entre cepillos Hilsdorf y finalmente entre placas de cartón o madera. El efecto de la sujeción de las placas sobre el pilar de cuatro unidades puede verse en las figuras 10 y 11, siguiendo las deformaciones verticales un esquema similar a las curvas de carga de las figuras 8 y 9. Para la única probeta ensayada la relación tensión/deformación verticales siguió una curva simple cuando el pilar se cargó entre placas de cartón o madera, mientras que las placas del cepillo de Hilsdorf produjeron un esquema tensión/deformación lineal. El módulo de Poisson era 0,138 cuando las probetas se cargaban entre cartones y 0,1105 entre cepillos de Hilsdorf.

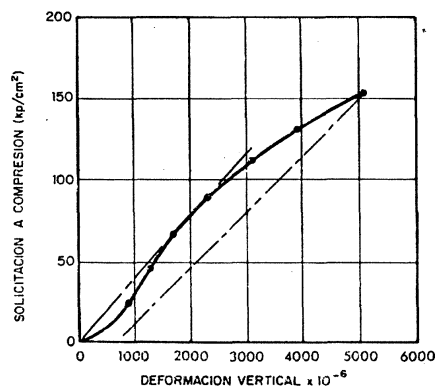

Fig. 10.-Medidas de deformación: pilar de fábrica de ladrillos entre placas de madera contrachapada.

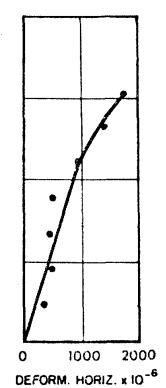

madera
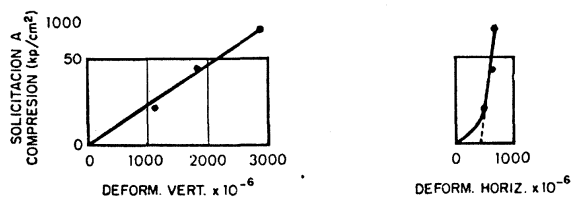

Fig. 11.-Medidas de deformación: pilar de fábrica de ladrillos entre cepillos Hilsdorf.

\section{VELOCIDAD DE CARGA - ENSAYO DE COMPRESION}

Los ensayos de velocidad de carga se llevaron a cabo sobre ladrillos silicocalcáreos: a) entre placas de contrachapado de caoba; b) entre placas de contrachapado de abedul; c) entre cepillos de Hilsdorf. El intervalo considerado oscilaba entre 4,344 y 26,062 $\mathrm{MN} / \mathrm{m}^{2} / \mathrm{min}$ y los resultados a compresión se publicaron en otra ocasión (3).

Los ensayos indicaron que al aumentar la velocidad de carga para probetas ensayadas entre madera contrachapada aumentaba la carga final en el intervalo 4,344 a 19,114 
$\mathrm{MN} / \mathrm{m}^{2} / \mathrm{min}$, pero entre 19,114 y $26,062 \mathrm{MN} / \mathrm{m}^{2} / \mathrm{min}$ se observaba una bajada. El contrachapado de caoba producía cargas finales de $1,379 \mathrm{MN} / \mathrm{m}^{2}$ mayores que la madera de abedul, en el intervalo más bajo, pero la diferencia en el límite de carga era sólo de $0,345 \mathrm{MN} / \mathrm{m}^{2}$.

Los ensayos de velocidad de carga empleando las placas del cepillo de Hilsdorf ilustran un fenómeno interesante: dentro de las velocidades de carga empleadas, cuando se eliminan las sujeciones laterales al emplear las placas de cepillo, la variación en la velocidad de carga crea poca diferencia en la resistencia a compresión de la unidad ensayada.

\section{MEDIDAS DE DEFORMACIONES DE PROBETAS DE HORMIGON Y DE RESINAS EPOXI}

La finalidad de esta experimentación era demostrar a los autores que los cepillos Hilsdorf se comportan satisfactoriamente como demostró Grimer (7). Se ensayaron dos probetas: una de hormigón $1: 2: 4(50 \times 20 \times 10 \mathrm{~cm})$ y otra de resina epoxi con una arena y filler PFA $(30 \times 23 \times 11 \mathrm{~cm})$. Las razones para la diferencia de tamaño de probetas eran:

a) Dos moldes de acero de $20 \times 10 \times 10 \mathrm{~cm}$ pueden empalmarse fácilmente en el primer caso para la probeta de hormigón.

b) Se eligieron específicamente las dimensiones $23 \times 11 \mathrm{~cm}$ para emparejar las dimensiones del cepillo, dictándose la altura máxima por la capacidad de la amasadora y facilidad de colocación de la muestra de una sola amasada.

Los ensayos se llevaron a cabo entre placas de acero y entre cepillos Hilsdorf. A pesar de que las placas del cepillo tenían dimensiones más parecidas a la probeta de resina epoxi que la probeta de hormigón, los mejores resultados se obtuvieron con el hormigón. Las deformaciones registradas en la cima de ambas probetas eran mayores que en la base, en parte debido a las condiciones finales menos estables y a la desigual retracción de las probetas.

La probeta de hormigón produjo el tipo de esquema de deformación anticipado (fig. 12). Contrariamente a lo esperado la probeta de epoxi señaló un esquema de deformación confuso, sugiriendo que la retracción y el método de fabricación produjeron una unidad que no debe ser menos homogénea necesariamente, pero donde se han producido tensiones que confunden los resultados.

La figura 12 demuestra que, dentro de los límites de errores experimentales, se elimina virtualmente la fijación lateral en la base de la probeta y ya se han explicado previamente las razones para la inconsistencia en la cima.

\section{RELACION RESISTENCIA LADRILLO/RESISTENCIA PARED EMPLEANDO LOS CEPILLOS DE HILSDORF}

Se llevaron a cabo ensayos para determinar si existe una relación constante entre la resistencia a compresión de los ladrillos ensayados entre cepillos de Hilsdorf y los paneles de pared de altura de pisos. En este campo se han hecho unos ensayos limitados y se espera continuar el trabajo. La relación de resistencia de pared a resistencia Hilsdorf, para los ensayos hasta ahora realizados, oscila de 0,534 a 0,89 . 


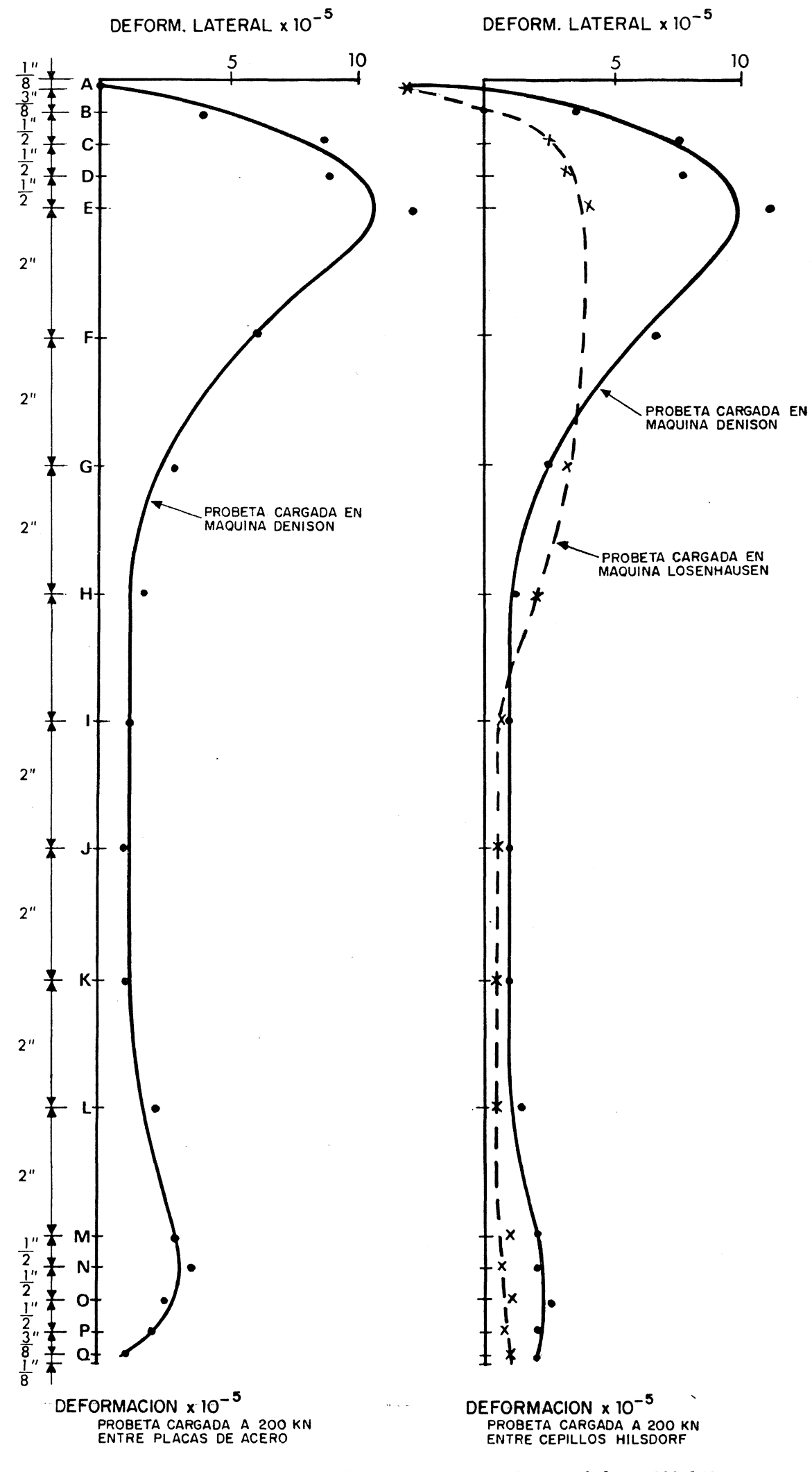

Fig. 12.-Deformaciones en probeta de hormigón a $200 \mathrm{kN}$. 


\section{R E S U M E N}

En 1969 se comenzó en la Universidad de Salford un programa de investigación para el ensayo de elementos estructurales de fábrica de ladrillo, bajo los auspicios de la Brick Development Association.

El trabajo iba dirigido a determinar la resistencia de los ladrillos bajo las tensiones a que ellos están sometidos en la práctica, que son predominantemente de tracción.

Este artículo compara y contrasta los diferentes ensayos de tracción que se investigaron, mientras que un tercer miembro del equipo de investigación describe, en el artículo que continúa, la aplicación de las técnicas de fotoelasticidad a este trabajo.

\section{BIBLIOGRAFIA}

(1) BSI: Structural Recommendations for Loadbearing Walls, CPIII, 1964.

(2) Thomas, K.: Structural Brickwork Materials and Strength. Thesis, MSc., University of Salford, 1970.

(3) Thomas, K., and O'LeARY, D. C.: Rate of loading tests on brick specimens to be published shortly.

(4) BSI: Specification for Bricks and Blocks of Fired Brickearth, Clay or Shale, BS3921: Part 2, 1969. Metric Units.

(5) Spetla, Z., and KADLECEK, V.: "Effects of Slenderness on the Direct Tensile Strength of Concrete Cylinders and Prisms". Bull. Rilem, núm. 33, December 1966.

(6) McNicholas, J. B.: Photoelastic Stress Analysis of Brick Masonry Systems. Second International Brick Masonry Conference, University of Keele, April 1970.

(7) BaLdWIN, L. W.: (Private Communication), BRS, October 1968. 